\title{
Letter to the editor, Odell 2019
}

\author{
Peter Tuchin*
}

Odell et al., should be commended on an important study for chiropractic treatment of chronic migraine [1]. However, in my opinion, there are some significant limitations in the design of the trial.

Firstly, with the trial having no placebo/sham group, the strength of the trial is decreased, and any potential conclusions will be significantly limited [2]. I suggest the authors consider adding a possible placebo/sham arm of the trial, which could include:

- Sham SMT [3]

- Detuned interferential/ultrasound [4]

- Placebo pharmaceutical, such as a "muscle relaxant"

- Detuned TENS or "Cefaly" (which is a brand name for an external trigeminal nerve stimulator) [5]

Alternatively, the authors could consider comparing mobilisation of the neck to SMT as separate cohorts, and not in the current method as a pragmatic study, where participants may receive either or both (dependent on practitioner discretion).

Secondly, I believe the study contains significant attention bias difference due to participants receiving five SMT sessions in comparison to no treatment sessions for care as usual (CAU) cohort. This will also potentially add to poor method scores in patient "blinding", as it should be obvious participants in the SMT cohort, that they have received therapeutic treatment, versus no treatment in the CAU cohort [2].

In addition, there are some concerns over the total number of outcome measures (ten) and poor compliance from participants due to the time to complete all outcome measures thoroughly. I wish the authors good luck in completion of this study.

\section{Response from the authors}

Jim Odell, Carol Clark, Adrian Hunnisett, Osman Hassan Ahmed and Jonathan Branney

The authors would like to thank Associate Professor Tuchin for taking the time to write to the editor about our protocol [1]. The issues raised are important and ones that we considered before undertaking the study.

With regard to the question on placebo arm, we accept that it may have been useful, although on the other hand introducing a valid placebo in any manual therapy randomised controlled trial (RCT) is difficult and rarely removes or accounts for all components of the placebo effect. However, we recognise some studies have attempted to do so $[6,7]$ and it is a future area for development.

In this study our objective was a pragmatic one, designed to aid real-world clinical decision making on treatment rather than understanding the causal nature of the relationship [8]. The design was therefore 'addon'. Some authors have suggested that the aim should be to identify the benefit to the patient regardless of the

\footnotetext{
* Correspondence: peter.tuchin@outlook.com

Macquarie University, North Ryde, Sydney, Australia
}

source; psychosocial or biological, particularly where risk to the patients is low $[9,10]$.

With regard to the suggestion of comparing mobilisation and spinal manipulative therapy (SMT) this is certainly a study that can be carried out in the future. We chose to explore the effectiveness of manual therapy (MT) (without specifying one type), as it was felt that this is what best mirrors clinical practice, and as such makes this a pragmatic trial.

We are unaware of the use of the specific term 'attention bias' but understand the point made. All participants were contacted every week for diary data, often with the non MT group more forthcoming, which may mitigate some of the suggested bias differential. However we are unaware of measures that quantify the impact of 'attention' bias. The 'blinding' however is an issue with this type of study and will be reflected in discussion of the methodological limitations rather than a score, which is not applicable to the CONSORT process $[11,12]$. 
The potential issues with compliance and outcome measures were considered and efforts made to mitigate these by involving people with chronic migraine in the design of the outcome measure booklets and in trialling the allocation of time to complete. We are able to report that our initial results indicate that the primary outcome measurement instruments were completed thoroughly. However, we noted that not all the migraine diaries were completed. This has provided us with valuable insights for further trials in this population.

\section{Author's contributions}

The author read and approved the final manuscript.

\section{Competing interests}

The author declares that he/she has no competing interests.

Received: 6 May 2019 Accepted: 19 July 2019

Published online: 20 August 2019

\section{References}

1. Odell J, Clark C, Hunnisett A, Ahmed OH, Branney J. Manual therapy for chronic migraine: a pragmatic randomised controlled trial study protocol. Chiropr Man Therap. 2019;27:11.

2. Turner $\mathrm{L}$, Shamseer $\mathrm{L}$, Altman DG, et al. Consolidated standards of reporting trials (CONSORT) and the completeness of reporting of randomised controlled trials (RCTs) published in medical journals. Cochrane Database Syst Rev. 2012;1:Mr000030.

3. Chaibi A, Benth JS, Tuchin PJ, Russell MB. Chiropractic spinal manipulative therapy for migraine: a three-armed, single-blinded, placebo, randomized controlled trial. Eur J Neurol. 2017;24:143-53.

4. Tuchin PJ, Pollard H, Bonello R. A randomized controlled trial of chiropractic spinal manipulative therapy for migraine. J Manip Physiol Ther. 2000;23:91-5.

5. Schoenen J, Vandersmissen B, Jeangette $S$, et al. Migraine prevention with a supraorbital transcutaneous stimulator: a randomized controlled trial. Neurology. 2013;80:697-704.

6. Chaibi A, ŠaltytèBenth J, Bjørn Russell M. Validation of placebo in a manual therapy randomized controlled trial. Sci Rep. 2015;5(1).

7. Cerritelli F, Ginevri L, Messi G, Caprari E, Di Vincenzo M, Renzetti C, Cozzolino V, Barlafante G, Foschi N, Provinciali L. Clinical effectiveness of osteopathic treatment in chronic migraine: 3-armed randomized controlled trial. Complement Ther Med. 2015;23(2):149-56.

8. Patsopoulos NA. A pragmatic view on pragmatic trials. Dialogues ClinNeurosci. 2011;13(2):217-24.

9. Vickers A. Why use placebos in clinical trials? A narrative review of the methodological literature. J Clin Epidemiol. 2000;53(2):157-61.

10. Avins A, Cherkin D, Sherman K, Goldberg H, Pressman A. Should we reconsider the routine use of placebo controls in clinical research? Trials. 2012;13(1).

11. Boutron I, Altman DG, Moher D, Schulz KF, Ravaud P, DJ C, et al. CONSORT statement for randomized trials of non pharmacologic treatments: a 2017 update and a CONSORT extension for non pharmacologic trial abstracts. Ann Intern Med Am Coll Physicians. 2017;167(1):40 PMID: 28630973.

12. Zwarenstein M, Treweek S, Gagnier JJ, Altman DG, Tunis S, Haynes B, Oxman AD, Moher D for the CONSORT and pragmatic trials in healthcare (Practihc) group. Improving the reporting of pragmatic trials: an extension of the CONSORT statement. BMJ. 2008;337:a2390 PMID: 19001484.

\section{Publisher's Note}

Springer Nature remains neutral with regard to jurisdictional claims in published maps and institutional affiliations. 